\title{
Radiation-Associated Lymphopenia and Outcomes of Patients with Unresectable Hepatocellular Carcinoma Treated with Radiotherapy
}

This article was published in the following Dove Press journal: Journal of Hepatocellular Carcinoma

Brian De (iD ${ }^{1} *$ Sweet Ping $\mathrm{Ng}^{1,2, *}$ Amy Y Liu' Santiago Avila (iD) 'Randa Tao ${ }^{3}$ Emma B Holliday' Zachary Brownlee ${ }^{4}$ Ahmed Kaseb ${ }^{5}$ Sunyoung Lee ${ }^{3}$ Kanwal Raghav $^{5}$ Jean-Nicolas Vauthey ${ }^{6}$ Bruce D Minsky' Joseph M Herman' Prajnan Das' Grace L Smith' Cullen M Taniguchi' Sunil Krishnan ${ }^{7}$ Christopher H Crane ${ }^{8}$ Clemens Grassberger 9 Theodore S Hong' Steven H Lin' Albert C Koong' Radhe Mohan' Eugene J Koay (ID)

'Department of Radiation Oncology, The University of Texas MD Anderson Cancer Center, Houston, TX, USA; ${ }^{2}$ Department of Radiation Oncology, Austin Health, Melbourne, Victoria, Australia; ${ }^{3}$ Department of Radiation Oncology, University of Utah, Huntsman Cancer Institute, Salt Lake City, UT, USA; ${ }^{4}$ Department of Radiation Oncology, Tufts Medical Center, Boston, MA, USA; ${ }^{5}$ Department of Gastrointestinal Medical Oncology, The University of Texas MD Anderson Cancer Center, Houston, TX, USA; ${ }^{6}$ Department of Surgical Oncology,

The University of Texas MD Anderson Cancer Center, Houston, TX, USA; ${ }^{7}$ Department of Radiation Oncology, Mayo Clinic Jacksonville, Jacksonville, FL, USA; ${ }^{8}$ Department of Radiation Oncology, Memorial Sloan Kettering Cancer Center, New York, NY, USA; ${ }^{9}$ Department of Radiation Oncology, Massachusetts General Hospital, Boston, MA, USA

*These authors contributed equally to this work

Correspondence: Eugene J Koay MD Anderson Cancer Center, Department of Radiation Oncology, 1400 Pressler St. Unit 1422, Houston, TX, 77030, USA

$\mathrm{Tel}+\mid$ 7|3-563-238|

Fax + | 7|3-563-233|

Email EKoay@mdanderson.org
Background: The immune system plays a crucial role in cancer surveillance. Previous studies have shown that lymphopenia associated with radiotherapy (RT) portends a poor prognosis. We sought to differentiate the effects of proton and photon RT on changes in absolute lymphocyte count (ALC) for patients with hepatocellular carcinoma (HCC).

Patients and Methods: Patients with HCC treated with definitive RT from 2006 to 2016 were studied. Serial ALCs were graded according to CTCAE v4.0. Overall survival (OS), disease-free survival, and distant metastasis-free survival were analyzed using the KaplanMeier method. Univariable and multivariable Cox-proportional hazards analyses were used to identify predictors of OS. A cohort analysis matched for treatment volume was performed to investigate differences in ALC dynamics between photon and proton therapy.

Results: Of 143 patients identified, the median age was 66 (range, 19-90) years. The treatment modality was photon in $103(72 \%)$ and proton in 40 (28\%). Median follow-up was 17 months (95\% confidence interval, 13-25 months). The median time to ALC nadir after initiation of RT was 17 days with a median relative decrease of $67 \%$. Those who received proton RT had a higher median ALC nadir $(0.41$ vs $0.32 \mathrm{k} / \mu \mathrm{L}, \mathrm{p}=0.002)$ and longer median OS (33 vs 13 months, $\mathrm{p}=0.002$ ) than those who received photon RT. Matched cohort analyses revealed a larger low-dose liver volume in the photon group, which correlated with lower ALC. On multivariable Cox analysis, Grade 3 or higher lymphopenia prior to or after RT, portal venous tumor thrombus, larger planning target volumes, Child-Pugh (CP) Class B, and increased CP score after RT were associated with a higher risk of death, whereas the use of proton therapy was associated with lower risk.

Conclusion: Grade 3 or higher lymphopenia may be associated with poorer outcomes in patients receiving RT for HCC. Protons may mitigate lymphopenia compared with photons, potentially due to reduced dose exposure of sites of lymphopoiesis.

Keywords: lymphocyte count, circulating lymphocytes, splenic dose, liver dose, overall survival

\section{Introduction}

Hepatocellular carcinoma (HCC) is the most common primary liver cancer and one of the leading causes of cancer death globally. ${ }^{1}$ The incidence of HCC is rapidly increasing and is disproportionately high in low- and middle-income countries and among groups of lower socioeconomic status in the United States. ${ }^{2}$ Despite recent advances in treatment, the 5-year overall survival for liver cancers remains around $20 \%{ }^{3}$ Advances in conformal photon and particle radiotherapy (RT) have bolstered the role of RT in the management of this disease. ${ }^{4}$ Conformal photon-based 
techniques such as intensity-modulated radiation therapy (IMRT) and volumetric modulated arc therapy (VMAT) have allowed clinicians to better optimize dose homogeneity at the expense of a larger volume of surrounding tissue receiving low doses of RT, termed the "low dose bath," which is hypothesized to increase the risk of toxicities or secondary malignancies. ${ }^{5,6}$ Through the use of proton RT, however, clinicians are better able to escalate doses to malignant tissues while sparing surrounding parenchyma thereby limiting the risk for worsening of liver function. ${ }^{4}$ Another potential benefit of proton RT is the reduced risk for high-grade lymphopenia. ${ }^{7-9}$ Previous studies have shown that high-grade lymphopenia portends poorer outcomes in patients with pancreatic, cervical, esophageal, head and neck, lung, and central nervous system malignancies. ${ }^{10-17}$ However, the effects of lymphopenia on the clinical course of patients treated with RT for unresectable HCC are not well characterized. Lower RT doses surrounding targets for the treatment of HCC may not directly injure organs at risk but may significantly deplete highly radiosensitive lymphocytes. ${ }^{18}$ This study aims to characterize the impact of treatment-related lymphopenia on outcomes in patients with HCC and elucidate the impact of proton and photon RT on lymphocyte counts and clinical outcomes.

\section{Methods}

\section{Patient Selection}

The Institutional Review Board Committee at MD Anderson Cancer Center approved our request to review the medical records of these patients. The need for informed patient consent was waived, as this was a retrospective review and no identifiable patient information is included in this report. This study was conducted in accordance with the ethical standards of the Declaration of Helsinki and its later amendments. We identified all patients aged $\geq 18$ years with unresectable HCC treated with definitive RT between 2006 and 2016 at MD Anderson Cancer Center. Patients who received doses $<30 \mathrm{~Gy}$, did not complete RT, did not have lymphocyte count data available at least prior to and during RT, and those with the distant disease at the time of treatment were excluded. Patient characteristics, tumor features, treatment parameters, and clinical outcomes were recorded. Child-Pugh scores (CPS) prior to RT and at peak within 9 months of RT completion were calculated to assess for liver decompensation, defined as an increase by $\geq 2$ points. Serial absolute lymphocyte counts (ALC) prior to, during, and after RT were collected and graded according to Common Terminology Criteria for Adverse Events (CTCAE) v4.0. Post-RT ALC data were recorded for patients with labs collected within 100 days of completing RT to capture patients returning for follow-up at approximately 3 months.

\section{Matched Cohort Analysis}

To investigate differences in ALC between treatment modalities, a cohort analysis of 15 proton and 15 photon cases matched to treatment volume was conducted. For each patient, body dose-volume indices $\mathrm{V}_{\mathrm{x}}$ (the volume receiving a dose of $\mathrm{X}$ or higher, eg, $\mathrm{V}_{1 \mathrm{~Gy}}, \mathrm{~V}_{5 \mathrm{G} y}, \mathrm{~V}_{10 \mathrm{~Gy}}$ ) were extracted from treatment planning software. Associations of various body low dose $\mathrm{V}_{\mathrm{x}}$ with treatment modality (protons vs photons) and ALC nadir, defined as the lowest lymphocyte count recorded during treatment, were investigated. Similarly, spleen volumes and mean doses were obtained from treatment plans and compared for the matched cohort analysis.

\section{Statistical Analysis}

Patient baseline characteristics were compared with $t$-tests for continuous variables and $\chi 2$ tests for categorical variables. ALC counts were used to determine the change from baseline and compared using the Wilcoxon signedrank test. Overall survival (OS), disease-free survival (DFS), and distant metastasis-free survival (DMFS) were measured from the start of RT, analyzed using the KaplanMeier method, and compared using the log-rank test. Correlation analysis with Bonferroni correction was performed using the Pearson method for continuous explanatory variables and the Spearman method for ordinal explanatory variables. Univariable and multivariable analyses were conducted using Cox proportional hazards. The proportional hazards assumptions were evaluated using a $\chi^{2}$ test of Schoenfeld residuals. To account for multicollinearity in the multivariable analysis, variable selection utilized stepwise elimination of the variable with the highest variance inflation factor (VIF) until the mean VIF of remaining variables was $\leq 2$; these variables comprised the final model. The multivariable analysis utilized timevarying covariates for post-treatment parameters. Continuous variables, including age, total RT dose, dose per fraction, time to ALC nadir, percent change from baseline in ALC at nadir, and change in CPS were included directly in univariable and multivariable models. Statistical analysis was performed with Stata Version 13.0 
(StataCorp, College Station, TX, USA) and JMP Version 14.0 (SAS Institute, Cary, NC, USA).

\section{Results}

\section{Patient Characteristics}

A total of 143 patients were included in this study. Patient, treatment, and tumor characteristics are summarized in Table 1 and also stratified by treatment technique for comparison. The median age at treatment was 66 (range, 19-90) years. The majority of the cohort was male (78\%) and of favorable performance status $(90 \%$ Eastern Cooperative Oncology Group [ECOG] 0-1). There were $103(72 \%)$ in the photon cohort and $40(28 \%)$ in the proton cohort. Among all patients, $40 \%$ had American Joint Committee on Cancer (AJCC) 8th Edition Stage I or II disease and $60 \%$ had Stage III or IV disease. This differed between treatment groups as a greater portion of protontreated patients had Stage I or II disease ( $65 \%$ vs $30 \%$; $\mathrm{p}<0.001)$. The Barcelona Clinic Liver Cancer stages were early stage (A) in $10(7 \%)$ patients, intermediate stage (B) in $2(1 \%)$, and advanced stage $(\mathrm{C})$ in $131(92 \%) .{ }^{19}$ Portal venous tumor thrombosis was present in 79 (55\%) patients; a greater proportion of photon-treated patients had portal venous thrombus present at the start of RT (62\% vs $38 \%$; $\mathrm{p}=0.008$ ). Child-Pugh A liver disease was noted in $114(80 \%)$ patients, including $80(56 \%)$ with A5 and 34 (24\%) with A6 disease. The mean pre-treatment Child-Pugh score was 5.8 (range, 5-9) for patients receiving photon RT and 5.8 (range, 5-8) for those receiving proton RT. The median prescribed dose was 60 Gy (range, 30-100 Gy, interquartile range [IQR], 50.4-67.5 Gy) with a median of 15 (range, 3-34, IQR, 15-25) fractions. The median prescribed dose per fraction was 3.9 Gy (range, 1.8-20 Gy). The median biologically equivalent dose (BED) was 84 Gy (range, 44-180 Gy) and 97 GyE (range, 39-140 GyE) for patients treated with photons and protons, respectively. Prior to RT a total of $64(45 \%)$ patients underwent one or more liver-directed local therapies: $56(39 \%)$ with transarterial chemoembolization (TACE), 9 (6\%) with radiofrequency ablation (RFA), 8 $(6 \%)$ with surgical resection, $5(3 \%)$ with portal vein embolization (PVE), and 4 (3\%) with Yttrium-90 (Y-90) radioembolization. Following RT, a total of 16 (11\%) patients underwent one or more liver-directed local therapies: $12(8 \%)$ with TACE, $5(3 \%)$ with a repeat course of RT, 3 (2\%) with ethanol ablation, and $1(1 \%)$ each with surgical resection, RFA, and Y-90 radioembolization.

\section{Changes in Absolute Lymphocyte Count During Radiotherapy}

The median pre-RT ALC was $1.10 \mathrm{k} / \mu \mathrm{L}$ (range, $0.11-3.79 \mathrm{k} /$ $\mu \mathrm{L})$ and 10 patients $(7 \%)$ had pre-RT ALC $\leq 0.5$. Post-RT ALC data within 100 days of completing RT are available for 131 patients $(92 \%)$. At a median of 42 days (range, 1-95 days, IQR, 24-60 days), the median post-RT ALC was 0.59 $\mathrm{k} / \mu \mathrm{L}$ (range, $0.03-2.12 \mathrm{k} / \mu \mathrm{L}$ ) and 59 patients $(45 \%)$ had post-RT ALC $\leq 0.5$. There was a significant decrease in ALC between pre-treatment and post-treatment periods (Figure 1A), with a median nadir reduction of $67 \%$ (IQR, 52-80\%). During treatment, $45 \%$ of patients developed Grade 3 (G3) lymphopenia and 22\% developed Grade 4 (G4) lymphopenia. Stratifying by technique, G3 or higher lymphopenia developed in $72 \%$ of patients receiving photons and $55 \%$ receiving protons $(\mathrm{p}=0.04)$ whereas $\mathrm{G} 4$ lymphopenia developed in $23 \%$ receiving photons and $8 \%$ receiving protons $(\mathrm{p}=0.007)$. The development of $\mathrm{G} 3$ or higher lymphopenia stratified by treatment technique is presented in Figure 1B. Univariable correlation analysis revealed an association between the percentage of ALC decrease during treatment and treatment modality (proton vs photon, $\mathrm{p}=0.04)$, pre-treatment ALC ( $\mathrm{p}=0.02)$, dose per fraction $(p=0.004)$, number of treatment fractions $(p=0.0002)$, and use of IMRT/VMAT vs 3D conformal RT ( $\mathrm{p}=0.03$ ). There was no correlation between the percentage of ALC decrease during treatment with age, sex, pre- or post-treatment ChildPugh score, change in Child-Pugh score, prior use of TACE, presence of portal vein tumor thrombus, total prescribed radiation dose, biologically effective dose (BED), volume of radiation target, or splenic volume.

As there was a significant correlation between pretreatment ALC and the percentage of ALC decrease during RT, we sought to identify factors that may influence pretreatment ALC. On univariable analysis, there was no correlation between pre-treatment ALC with age, presence of portal vein tumor thrombosis, or pre-treatment ChildPugh score, but there was a significant inverse association with increasing splenic volume $(\mathrm{r}=-0.45, \mathrm{p}<0.0001)$.

\section{Overview of Clinical Outcomes}

At a median follow-up of 16.7 months (range, 1-117 months), $29 \%$ of all patients were alive at the last follow-up. Recurrences were local in $17 \%$ at median 8.5 months (95\% CI, 3.8-11.9 months), out-of-field liver only in 39\% at median 6.3 months (95\% CI 5.1-8.2), and distant metastasis in 31\% at median 6.1 months (95\% CI, 3.9-7.4 months). After RT, the 
Table I Baseline Patient, Tumor and Treatment Characteristics

\begin{tabular}{|c|c|c|c|c|}
\hline Parameter & All Patients $(n=\mid 43)$ & $\begin{array}{l}\text { Photon-Treated Patients } \\
(n=103)\end{array}$ & $\begin{array}{l}\text { Proton-Treated Patients } \\
(n=40)\end{array}$ & p-value \\
\hline Median age (range) & $66(19-90)$ & $63(19-90)$ & $73(5 I-89)$ & $<0.001$ \\
\hline $\begin{array}{l}\text { Sex } \\
\qquad \text { Male } \\
\text { Female }\end{array}$ & $\begin{array}{l}\text { III (78\%) } \\
32(22 \%)\end{array}$ & $\begin{array}{l}82(80 \%) \\
21(20 \%)\end{array}$ & $\begin{array}{l}29(73 \%) \\
\text { II (28\%) }\end{array}$ & 0.360 \\
\hline $\begin{array}{c}\text { ECOG } \\
0 \\
1 \\
2\end{array}$ & $\begin{array}{l}27(19 \%) \\
101(7 \mid \%) \\
15(10 \%)\end{array}$ & $\begin{array}{l}21(20 \%) \\
85(72 \%) \\
8(8 \%)\end{array}$ & $\begin{array}{l}6(15 \%) \\
27(68 \%) \\
7(18 \%)\end{array}$ & 0.210 \\
\hline $\begin{array}{l}\text { AJCC 8th Edition Stage } \\
\text { I-II } \\
\text { III-IV }\end{array}$ & $\begin{array}{l}57(40 \%) \\
86(60 \%)\end{array}$ & $\begin{array}{l}31(30 \%) \\
72(70 \%)\end{array}$ & $\begin{array}{l}26(65 \%) \\
14(35 \%)\end{array}$ & $<0.001$ \\
\hline $\begin{array}{l}\text { Median baseline ALC in } \mathrm{k} / \mu \mathrm{L} \\
\text { (range) }\end{array}$ & $1.10(0.11-3.79)$ & $1.09(0.37-3.79)$ & $1.23(0.1 \mathrm{I}-2.83)$ & 0.925 \\
\hline $\begin{array}{l}\text { Pre-treatment Child-Pugh score } \\
\text { A5 } \\
\text { A6 } \\
\text { B7 } \\
\text { B8 } \\
\text { B9 }\end{array}$ & $\begin{array}{l}80(56 \%) \\
34(24 \%) \\
18(13 \%) \\
10(7 \%) \\
1(1 \%)\end{array}$ & $\begin{array}{l}55(54 \%) \\
26(25 \%) \\
14(14 \%) \\
6(6 \%) \\
1(1 \%)\end{array}$ & $\begin{array}{l}24(60 \%) \\
8(20 \%) \\
4(10 \%) \\
4(10 \%) \\
0(0 \%)\end{array}$ & 0.745 \\
\hline $\begin{array}{l}\text { Portal venous tumor } \\
\text { thrombosis } \\
\text { Yes } \\
\text { No }\end{array}$ & $\begin{array}{l}79(55 \%) \\
64(45 \%)\end{array}$ & $\begin{array}{l}64(62 \%) \\
39(38 \%)\end{array}$ & $\begin{array}{l}15(38 \%) \\
25(63 \%)^{*}\end{array}$ & 0.008 \\
\hline $\begin{array}{l}\text { Median splenic volume (IQR)** } \\
\text { Median dose (range) } \\
\text { Median \# of fractions (range) } \\
\text { Median planning target volume } \\
\text { (IQR) }\end{array}$ & $\begin{array}{l}322 \mathrm{~cm}^{3}\left(|97-55| \mathrm{cm}^{3}\right) \\
60 \mathrm{~Gy}(30-100 \mathrm{~Gy}) \\
15(3-34) \\
297 \mathrm{~cm}^{3}\left(|43-54| \mathrm{cm}^{3}\right)\end{array}$ & $\begin{array}{l}333 \mathrm{~cm}^{3}\left(219-55 \mathrm{Icm}^{3}\right) \\
60 \mathrm{~Gy}(35-100 \mathrm{~Gy}) \\
\mathrm{I} 5(3-34) \\
286 \mathrm{~cm}^{3}\left(129-529 \mathrm{~cm}^{3}\right)\end{array}$ & $\begin{array}{l}248 \mathrm{~cm}^{3}\left(187-537 \mathrm{~cm}^{3}\right) \\
68 \mathrm{GyE}(30-100 \mathrm{GyE}) \\
15(10-30) \\
339 \mathrm{~cm}^{3}\left(177-573 \mathrm{~cm}^{3}\right)\end{array}$ & $\begin{array}{l}0.328 \\
0.050 \\
0.343 \\
0.491\end{array}$ \\
\hline
\end{tabular}

Notes: Attributes for photon-treated and proton-treated patients are compared with $t$-test for continuous variables and $\chi 2$ test for categorical variables. Significant differences between patients treated with photons and protons are highlighted in bold. *Percentages do not sum to $100 \%$ due to rounding. **Spleen volumes were available for 108 patients (76\%), including 73 (7I\%) treated with photons and 35 (88\%) treated with protons.

Abbreviations: IQR, interquartile range; AJCC, American Joint Commission on Cancer; ECOG, Eastern Cooperative Oncology Group; ALC, absolute lymphocyte count.

mean change in Child-Pugh score was +1.5 (95\% CI, 1.1-1.8). A worsening of Child-Pugh score by $\geq 2$ developed in 42 patients (29\%). Among all patients, the median OS was 13.8 months (95\% CI, 11.1-16.7 months), the median DFS was 7.4 months (95\% CI, 5.4-8.8 months), and the median DMFS was 30.0 months (95\% CI, 20.6-45.7).

Factors on univariable analysis significantly associated with worse overall survival (Table 2) included the presence of portal venous tumor thrombus, increasing pre-RT ChildPugh score, pre-RT ALC $\leq 0.5$, post-RT ALC $\leq 0.5$, G3 or higher lymphopenia during treatment, use of IMRT/VMAT, increasing volume of planning target volume (PTV), and increase in Child-Pugh score after RT. Factors significantly associated with improved OS included increasing total RT dose and the use of proton therapy.

Variable selection for the multivariable model began with all variables shown in Table 2. Stepwise selection yielded a mean VIF of 1.74; variables in the final multivariable model included are listed in Table 3. The final model included 110 patients to account for patients with one or more missing values. A test of the proportional hazards assumption yielded $\chi^{2}(19, \mathrm{n}=110)=16.62, \mathrm{p}=0.616$ and thus the null hypothesis that the hazards are proportional cannot be rejected. The presence of portal venous tumor thrombus, Child-Pugh Class B, pre-RT ALC $\leq 0.5$, post-RT ALC $\leq 0.5$, increasing volume of PTV, and increased Child-Pugh score after RT were independently 


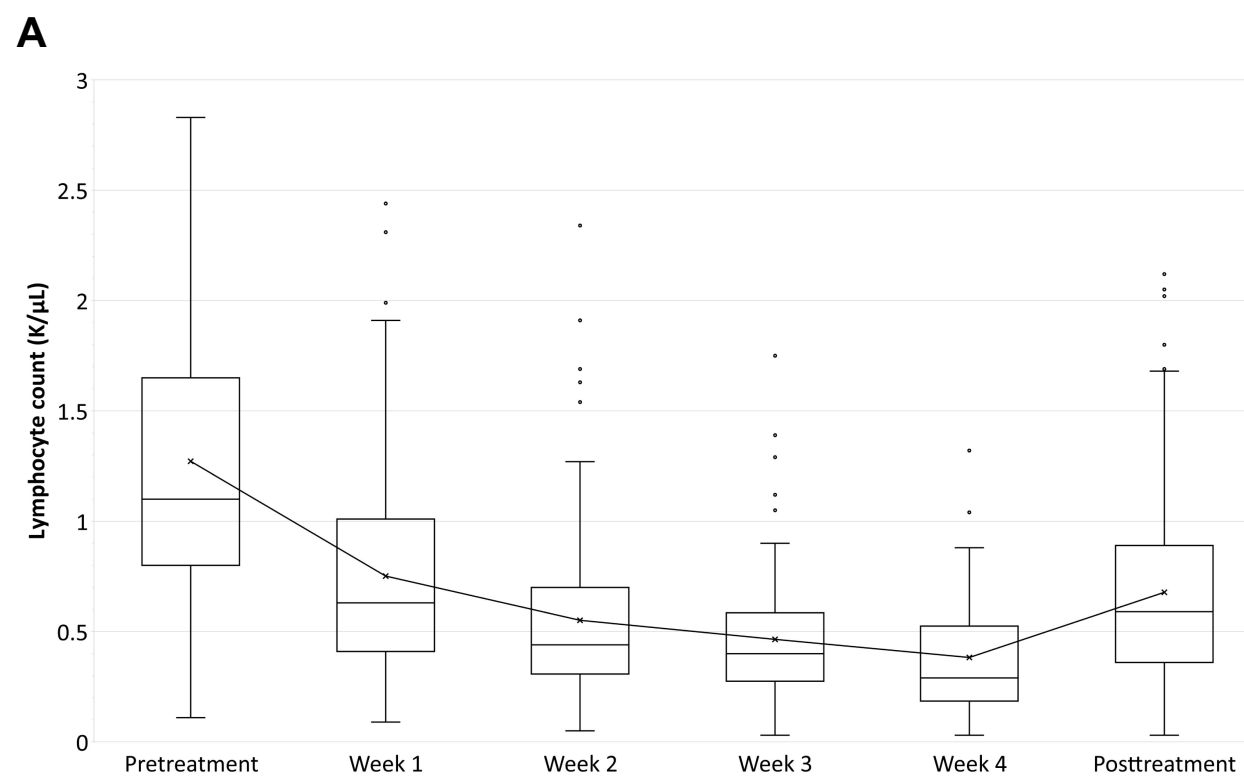

\section{B}

$100 \%$

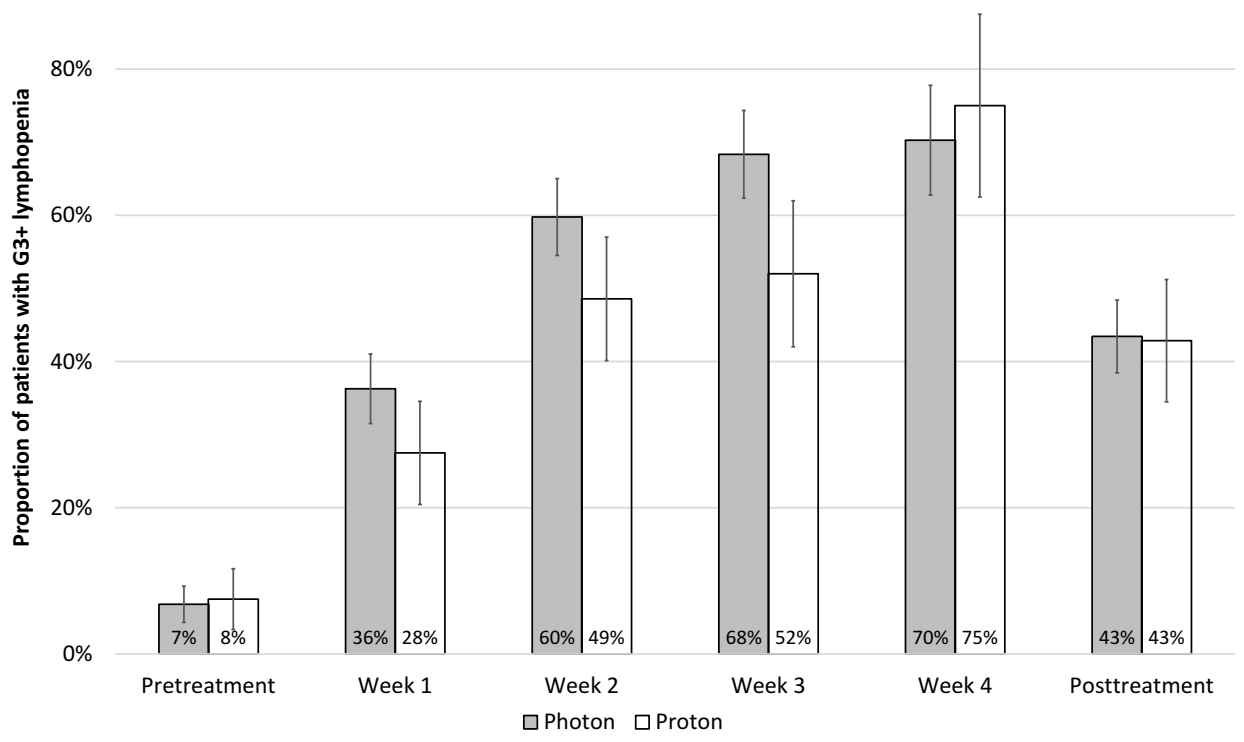

Figure I (A) The kinetics of lymphocyte counts during radiotherapy and (B) the proportion of patients developing G3 or higher lymphopenia stratified by treatment technique.

associated with a significant increased hazard of death, whereas the use of proton RT was independently associated with a reduced risk of death.

\section{Relationship Between Grade 3 or Higher Lymphopenia and Clinical Outcomes}

Patients with pre-RT ALC $\leq 0.5$ had significantly shorter OS (median 7 vs 20 months, $\mathrm{p}=0.03$ ) and DFS (median 7 vs 28 months) but not DMFS (11 vs 30 months, $\mathrm{p}=0.71$ ). Patients who had G3 or higher lymphopenia during RT had inferior OS (median 13 vs 31 months, $\mathrm{p}<0.001$ ) and DMFS (median 16 vs 51 months, $\mathrm{p}=0.003$ ) but not DFS (median 25 vs 37 months, $\mathrm{p}=0.0502$ ) compared with their counterparts (Figure 2). Splenic volume did not correlate with OS or DMFS. G3 or higher lymphopenia during treatment was significantly associated with the inferior OS on univariate analysis. On multivariable analysis, $\mathrm{ALC} \leq 0.5$ 
Table 2 Univariable Analysis for Overall Survival

\begin{tabular}{|c|c|c|c|}
\hline Parameters & Hazard Ratio & 95\% Confidence Interval & p-value \\
\hline Age & 0.998 & $1.019-1.086$ & 0.002 \\
\hline \multicolumn{4}{|l|}{ Sex } \\
\hline Female & (Reference group) & & \\
\hline Male & 1.017 & $0.643-1.610$ & 0.942 \\
\hline \multicolumn{4}{|l|}{ AJCC Stage } \\
\hline 1 & (Reference group) & & \\
\hline II & 1.055 & $0.534-2.085$ & 0.878 \\
\hline IIIA & 1.803 & $0.74 \mid-4.386$ & 0.194 \\
\hline IIIB & 1.448 & $0.884-2.372$ & 0.141 \\
\hline IVA & 1.272 & $0.629-2.573$ & 0.504 \\
\hline IVB & 1.660 & $0.720-3.824$ & 0.234 \\
\hline \multicolumn{4}{|l|}{ ECOG Performance Status } \\
\hline 0 & (Reference group) & & \\
\hline 1 & 1.460 & $0.825-2.584$ & 0.194 \\
\hline 2 & 1.532 & $0.706-3.321$ & 0.280 \\
\hline Portal venous tumor thrombus present & $1.55 \mathrm{I}$ & $1.043-2.305$ & 0.030 \\
\hline \multicolumn{4}{|l|}{ Pre-RT Child-Pugh Class } \\
\hline A & (Reference group) & & \\
\hline B & 1.846 & $1.164-2.929$ & 0.009 \\
\hline Viral hepatitis etiology & 0.837 & $0.563-1.244$ & 0.378 \\
\hline Prior transarterial chemoembolization & 0.742 & $0.493-1.115$ & 0.151 \\
\hline Pre-RT Child-Pugh score $>5$ & 1.691 & $1.138-2.513$ & 0.009 \\
\hline Pre-RT ALC $\leq 0.5$ & 2.096 & $1.052-4.177$ & 0.035 \\
\hline Post-RT ALC $\leq 0.5$ & $1.68 \mathrm{I}$ & $1.119-2.525$ & 0.012 \\
\hline Time to ALC nadir & 1.020 & $0.999-1.041$ & 0.059 \\
\hline$\%$ change from baseline in ALC at nadir & 1.634 & $0.599-4.460$ & 0.338 \\
\hline Grade $\geq 3$ lymphopenia during RT & 2.201 & $1.380-3.5 \mathrm{II}$ & 0.001 \\
\hline Use of breath hold & 1.135 & $0.763-1.688$ & 0.531 \\
\hline Use of IMRT/VMAT & 1.680 & $1.110-2.542$ & 0.014 \\
\hline Use of proton therapy & 0.498 & $0.315-0.786$ & 0.003 \\
\hline Total RT dose (Gy) & 0.978 & $0.96 I-0.995$ & 0.012 \\
\hline Dose per fraction (Gy) & 0.947 & $0.86 I-1.041$ & 0.258 \\
\hline Volume of PTV (in cc) & 1.0001 & $1.0000-1.0001$ & 0.001 \\
\hline Change in Child-Pugh score after RT & 1.765 & $1.541-2.022$ & $<0.001$ \\
\hline
\end{tabular}

Note: Significant associations are highlighted in bold.

Abbreviations: AJCC, American Joint Commission on Cancer; ECOG, Eastern Cooperative Oncology Group; ALC, absolute lymphocyte count; IMRT, intensity-modulated radiation therapy; VMAT, volumetric modulated arc therapy; PTV, planning target volume.

prior to or after RT - but not during - was found to be significantly associated with inferior OS. Overall, those who received proton RT had a higher median ALC nadir ( 0.41 vs $0.32 \mathrm{k} / \mu \mathrm{L}, \mathrm{p}=0.002$ ) than those who had photon $\mathrm{RT}$.

\section{Relationship Between Splenic Volume and Lymphopenia}

The development of lymphopenia is often multifactorial, and in this population of HCC patients treated with radiotherapy, one potential confounder may be splenomegaly secondary to portal hypertension. To further explore this, we measured the splenic volume on planning $\mathrm{CT}$ and found that splenic volume correlated with the development of G3 or higher lymphopenia $(\mathrm{p}<0.001)$ during RT (Figure $3 \mathrm{~A})$. To investigate whether there were treatment-related effects on lymphocytes and patient outcomes, we compared the splenic volumes of those treated with photons to those who had proton therapy. As shown in Figure 3B, there was no 
Table 3 Multivariable Analysis for Overall Survival Using Selected Variables After Adjustment for Multicollinearity

\begin{tabular}{|c|c|c|c|}
\hline Parameters & Hazard Ratio & 95\% Confidence Interval & p-value \\
\hline Age & 1.043 & $1.013-1.074$ & 0.004 \\
\hline \multicolumn{4}{|l|}{ Sex } \\
\hline Female & (Reference group) & & \\
\hline Male & 0.756 & $0.399-1.43 \mid$ & 0.391 \\
\hline \multicolumn{4}{|l|}{ AJCC Stage } \\
\hline 1 & (Reference group) & & \\
\hline II & 1.234 & $0.468-3.256$ & 0.671 \\
\hline IIIA & 0.882 & $0.24 I-3.220$ & 0.849 \\
\hline IIIB & 0.447 & $0.153-1.306$ & 1.141 \\
\hline IVA & 0.542 & $0.174-1.679$ & 0.288 \\
\hline IVB & 2.171 & $0.642-7.344$ & 0.213 \\
\hline \multicolumn{4}{|l|}{ ECOG Performance Status } \\
\hline 0 & (Reference group) & & \\
\hline 1 & 0.963 & $0.462-2.007$ & 0.920 \\
\hline 2 & 0.831 & $0.282-2.454$ & 0.739 \\
\hline Portal venous thrombus present & 3.576 & $1.472-8.683$ & 0.005 \\
\hline \multicolumn{4}{|l|}{ Pre-RT Child-Pugh Class } \\
\hline A & (Reference group) & & \\
\hline B & 2.330 & $1.04 I-5.210$ & 0.039 \\
\hline Pre-RT Child-Pugh score $>5$ & 0.889 & $0.442-1.785$ & 0.740 \\
\hline Pre-RT ALC $\leq 0.5$ & 2.677 & I.057-6.779 & 0.039 \\
\hline *Post-RT ALC $\leq 0.5$ & 1.031 & $1.001-1.062$ & 0.043 \\
\hline Total RT dose (Gy) & 0.9999 & $0.9996-1.0001$ & 0.471 \\
\hline Volume of PTV (cc) & 1.001 & $1.000-1.001$ & $<0.001$ \\
\hline *Change in Child-Pugh score after RT & 1.037 & $1.021-1.053$ & $<0.001$ \\
\hline Time to ALC nadir (days) & 1.010 & $0.979-1.042$ & 0.526 \\
\hline Use of proton RT & 0.456 & $0.236-0.88 I$ & 0.019 \\
\hline
\end{tabular}

Notes: Variables specified as time-varying are indicated by *. Significant associations are highlighted in bold.

Abbreviations: AJCC, American Joint Commission on Cancer; ECOG, Eastern Cooperative Oncology Group; ALC, absolute lymphocyte count; PTV, planning target volume.

significant difference in the splenic volume between the two groups.

\section{Outcomes Stratified by Treatment Technique}

After RT, the mean change in Child-Pugh score was $+1.8(95 \%$ confidence interval [CI], 1.3-2.2) for photon patients vs $+0.8(95 \% \mathrm{CI}, 0.3-1.2)$ for proton patients ( $\mathrm{p}=0.006)$. A worsening of Child-Pugh score by $\geq 2$ developed in $34 \%$ of photon patients and $18 \%$ of proton patients $(\mathrm{p}=0.03)$. The median OS with photon RT was 13.2 months (95\% CI, 9.6-16.7 months) and with proton RT was 33.2 months (95\% CI, 19.8-50.7 months). The median DFS with photon RT was 19.6 months (95\% CI, 12.7-28.0 months) and with proton
RT was 36.2 months (95\% CI, 29.2-56.0 months). Median DMFS with photon RT was 45.7 months (95\% CI, 11.5 months - upper bound undefined) vs 30 months for proton RT (95\% CI, 21.7-42.5 months). Kaplan-Meier survival curves for OS and DFS stratified by treatment technique are displayed in Figure 2.

\section{Matched Cohort Dosimetric Analyses of Proton versus Photon Cases}

Matched cohort dosimetric analyses revealed that there were significantly higher body volumes of low-dose bath in the photon group $\left(\mathrm{V}_{1 \mathrm{~Gy}}\right.$ and $\mathrm{V}_{10 \mathrm{~Gy}}, \mathrm{p}<0.0001$ for both; $\mathrm{V}_{5 \mathrm{~Gy}}, \mathrm{p}=0.002$ ) compared to the proton group (Figure 4). Higher body volume of low-dose bath correlated with lower ALC $\left(r=-0.34, p=0.06\right.$ for $\mathrm{V}_{1 \mathrm{~Gy}} ; \mathrm{r}=$ 
A
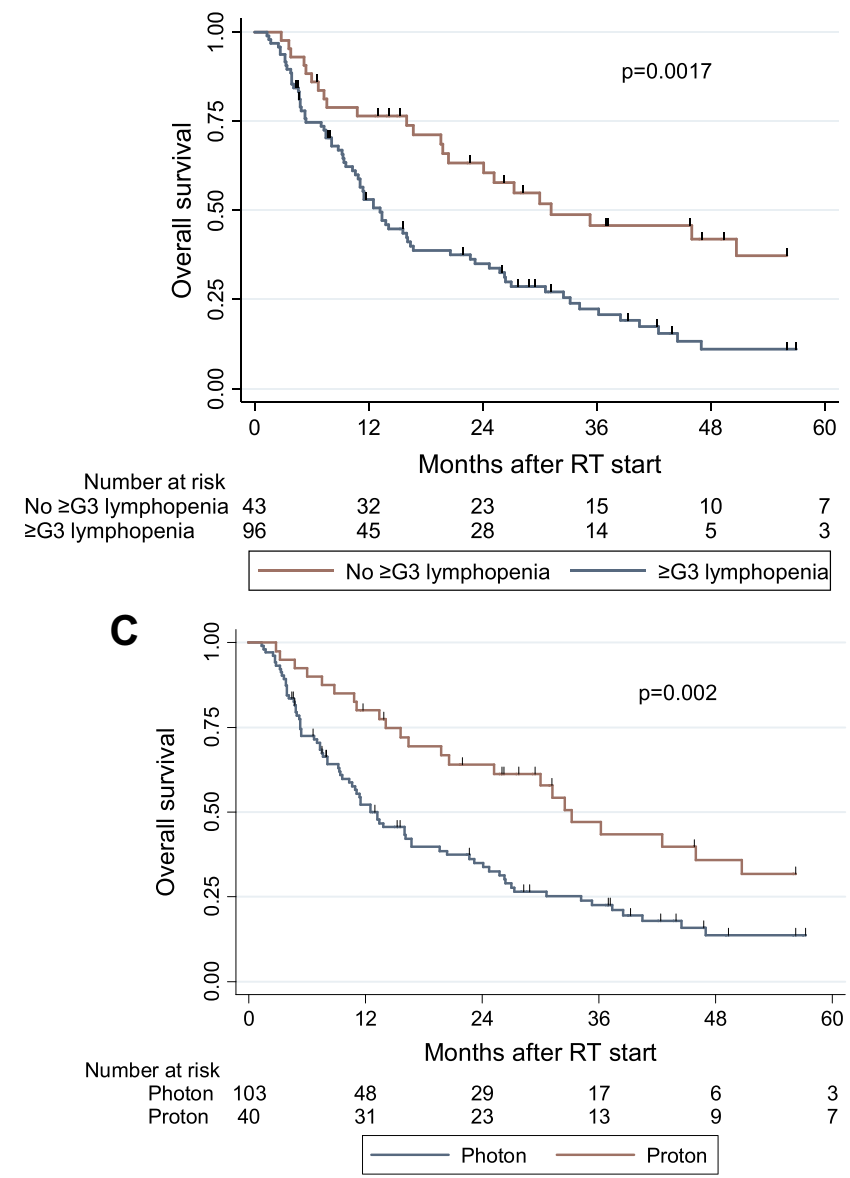

B

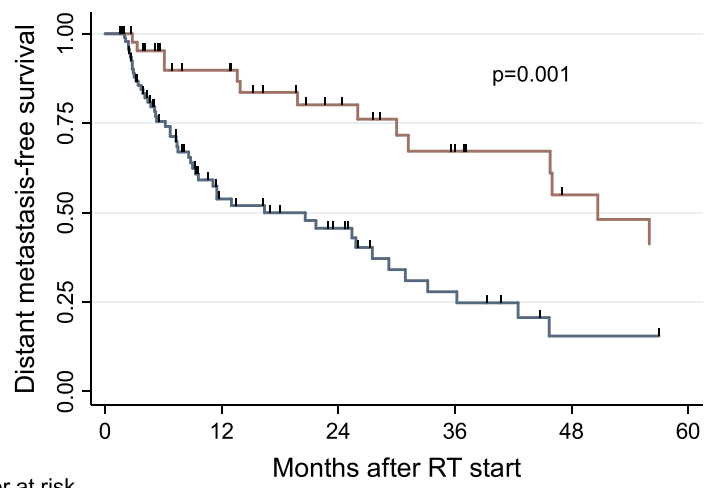

Number at risk \begin{tabular}{lllllll} 
No $\geq$ G3 lymphopenia & 43 & 31 & 21 & 13 & 8 & 6 \\
$\geq$ G3 lymphopenia & 96 & 29 & 19 & 9 & 3 & 2 \\
\hline
\end{tabular}
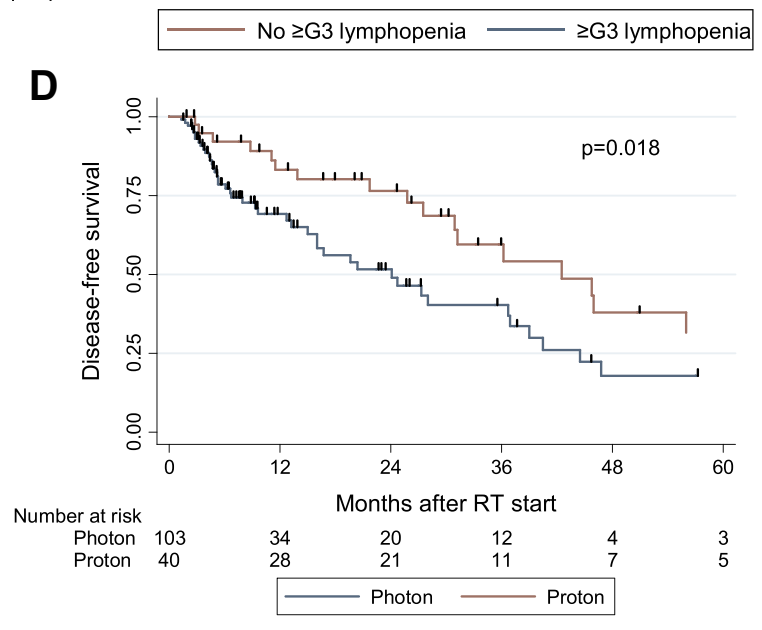

Figure 2 Survival outcomes stratified by development of grade 3 or higher lymphopenia after RT start (A and B) and treatment technique (C and D).
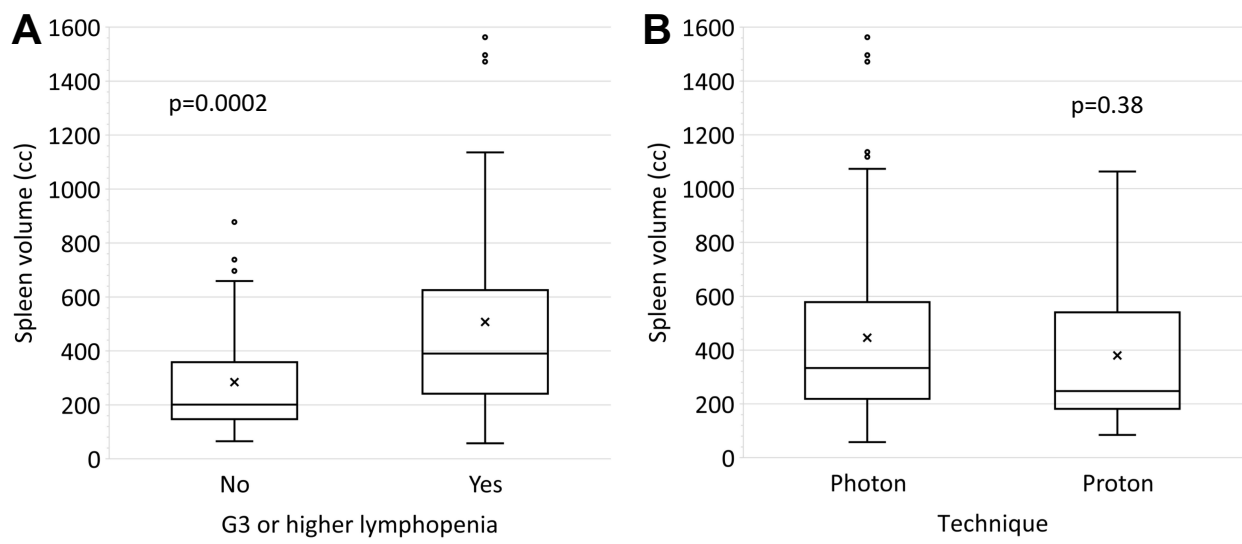

Figure 3 Distribution of splenic volumes stratified by (A) the development of G3 or higher lymphopenia during treatment and (B) photon vs proton therapy. Spleen volumes were available for 108 patients $(76 \%)$, including $73(71 \%)$ treated with photons and $35(88 \%)$ treated with protons.

\section{$-0.44, \mathrm{p}=0.01$ for $\mathrm{V}_{5 \mathrm{~Gy}} ; \mathrm{r}=-0.51, \mathrm{p}=0.004$ for $\left.\mathrm{V}_{10 \mathrm{~Gy}}\right)$. Discussion}

In addition, splenic irradiation dose for the paired In the current study, we demonstrate that patients with cohort was correlated with ALC nadir, and proton cases had significantly less splenic radiation dose $(\mathrm{p}<0.0001)$. unresectable HCC who developed G3 or higher radiation-related lymphopenia had significantly poorer OS and DMFS than those who did not. The presence of G3 
A

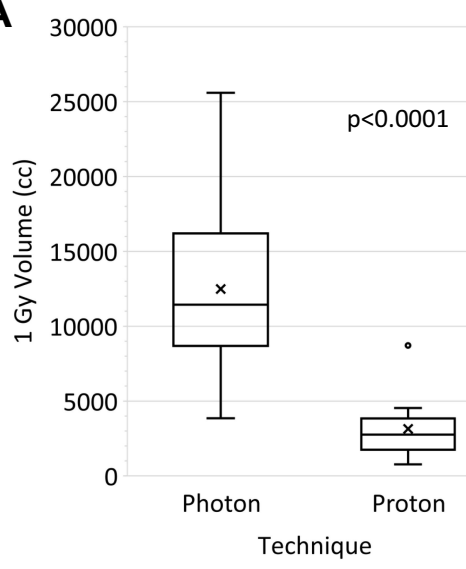

B

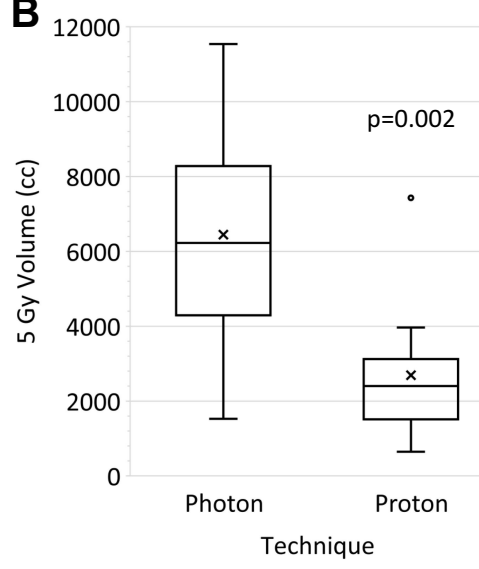

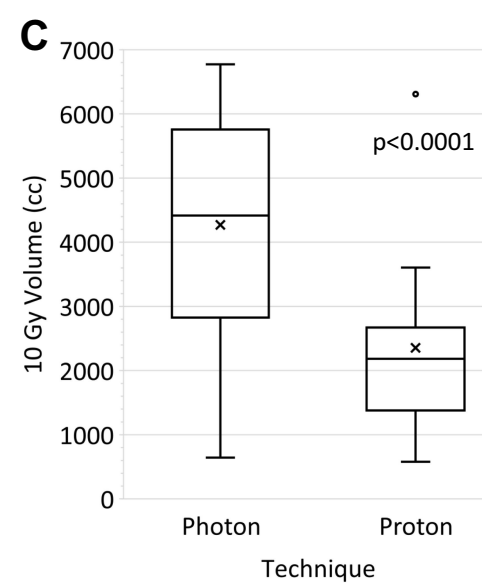

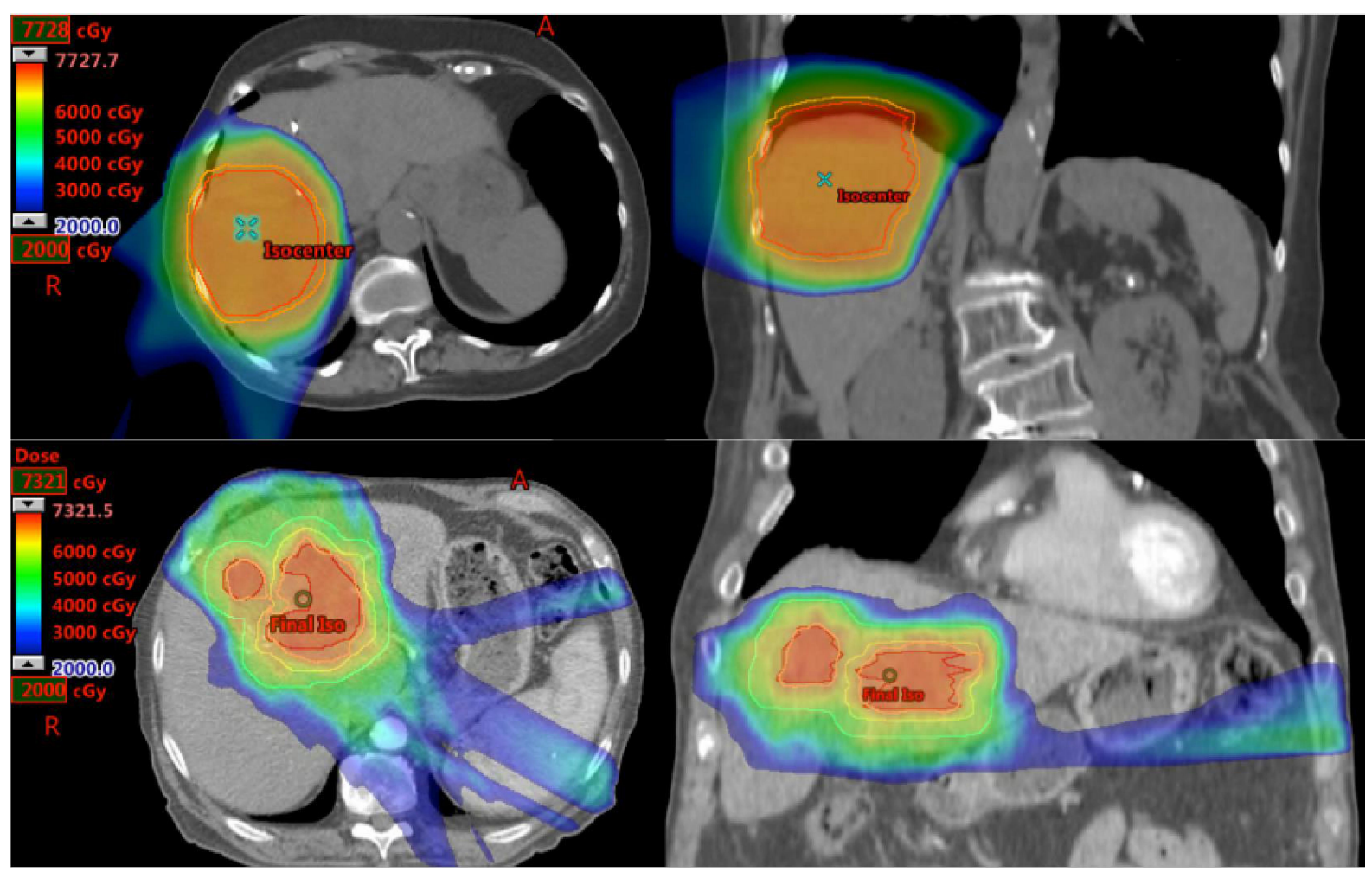

Figure 4 Matched cohort dosimetric analysis comparing volumes between photon versus proton groups receiving (A) I Gy, (B) 5 Gy, and (C) I0 Gy. A representative comparison of proton (top panel) and photon (bottom panel) treatment plans is shown.

or higher lymphopenia before the start of RT and after $\mathrm{RT}$ completion were found to be independent predictors of poorer survival. While G3 or higher lymphopenia can develop during both photon- and proton-based RT, proton therapy was associated with significantly lower rates of high-grade lymphopenia. In addition, on matched cohort analysis, our analysis demonstrated that proton-treated patients had significantly less lowdose radiation volume and splenic irradiation, which may help to explain the lower rates of high-grade lymphopenia in this cohort.
Radiation-associated lymphopenia has been associated with poorer patient outcomes in a variety of cancer types. A combined cohort of various newly diagnosed solid tumors demonstrated that high-grade lymphopenia was associated with an increased risk of death in all pathology types studied (hazard ratio [HR] 2.1, 95\% CI, 1.54-2.78; $\mathrm{p}<0.0001) .{ }^{20} \mathrm{~A}$ study at the MD Anderson Cancer Center demonstrated the adverse effects of high-grade lymphopenia on outcomes in patients with esophageal cancer. ${ }^{21}$ In another study of patients treated for non-small cell lung cancer, lower lymphocyte counts during RT correlated 
with larger gross tumor volumes and significantly reduced OS (HR 0.51 per $10^{3}$ lymphocytes $/ \mu \mathrm{L} ; \mathrm{p}=0.01$ ). ${ }^{14} \mathrm{In}$ a study of limited-stage small cell lung cancer, median OS was found to be 19 months in the lymphopenia group and 132 months in the group that did not develop high-grade lymphopenia. ${ }^{15}$ Among patients treated with RT for locally advanced rectal cancer, those with higher ALC nadir had improved response rates $(89.7 \%$ vs $67.6 \%$, $\mathrm{p}=0.006$ ) and OS (HR 0.24, CI, 0.08-0.69, $\mathrm{p}=0.004) .{ }^{22}$ In a cohort of patients with HCC treated with RT, patients with high-grade lymphopenia had decreased OS as compared to those without lymphopenia (13.6 vs 46.7 months, $\mathrm{p}<0.001){ }^{23}$ Further studies of the effects of radiation therapy in HCC have characterized the effects of RT fractionation on lymphocyte depletion and the relevance of specific T-cell populations in antitumor immunity. ${ }^{18,24}$ Overall, these previous studies are consistent with our current findings that G3 or higher lymphopenia was associated with poorer OS (13 vs 31 months).

The mechanism by which lymphopenia develops during RT is hypothesized to be due to the effect of the integral radiation dose on the regional lymph nodes, neighboring organs, and circulating blood volume, suggesting a benefit for use of protons over photons. Indeed, we demonstrated a reduced low-dose bath with protons as compared to photons in a matched cohort analysis. The suggested lymphocyte-sparing capabilities of proton RT in the present study are in line with what previous studies have found in other malignancies. An esophageal cancer study found that $40.4 \%$ of patients treated with photons developed grade 4 lymphopenia compared to $17.6 \%$ in the proton group $(\mathrm{p}<0.0001) .{ }^{9}$ Similarly, a propensity-matched cohort analysis conducted at the Mayo Clinic found that photon RT was associated with higher rates of G4 lymphopenia than proton RT in patients with esophageal cancer $(56 \%$ vs $22 \%$; $<<0.01) .{ }^{25}$ Additionally, a propensitymatched analysis of 144 patients treated with proton RT or photon RT for esophageal cancer demonstrated that photon RT resulted in higher rates of high-grade lymphopenia as compared to proton RT $(56 \%$ vs $22 \%$; p $<0.01) .{ }^{25}$ Previous studies have explored other RT modalities, such as stereotactic body radiotherapy (SBRT) as a means of mitigating lymphopenia. SBRT has been associated with a lower risk for lymphopenia in treating pancreatic ductal adenocarcinoma and has been shown to have a normal liver-sparing effect for smaller $\mathrm{HCC}$ lesions. ${ }^{26}$ Hypofractionated treatment in HCC has been shown to lead to decreased lymphocyte depletion and faster recovery following treatment. ${ }^{18}$

Several studies have shown encouraging data for immunotherapy in the treatment of HCC, either as monotherapy or in combination with other therapies, such as targeted agents or RT. ${ }^{27}$ However, the impact of lymphopenia on these novel agents and combinations remains a nascent area of investigation. A recent retrospective study of advanced non-small cell lung cancer demonstrated that peri-immunotherapy lymphopenia was associated with significantly poorer PFS and OS, and that multiple courses, multiple irradiated sites, and higher dose were correlated with RT-associated lymphopenia. ${ }^{28}$ Similarly, an analysis of Phase I/II lung cancer trials showed that SBRT was associated with an attenuated decrease in ALC in patients receiving combined immunotherapy with $\mathrm{RT}^{29}$ The impact of lymphopenia is particularly important to consider when deciding upon RT target volume and technique; in addition to its direct association with poorer outcomes, treatment-related lymphopenia may potentially limit the efficacy of promising immunotherapy agents in HCC. ${ }^{24}$ In this context, further investigations into optimal selection, sequencing, and timing of treatments, as well as therapeutic strategies to enable recovery of lymphocytes, are needed. ${ }^{30}$

Our data show that proton RT was significantly associated with improved OS and DFS. The OS benefit was also demonstrated on multivariable analysis, which showed that the use of proton therapy was independently associated with a decreased risk of death by more than half. Data from other institutions are concordant with our findings. The Massachusetts General Hospital reported that proton therapy was associated with a decrease in the development of the non-classic radiation-induced liver disease, as defined by an increase in baseline CP score by $\geq 2$ points at 3 months posttreatment. In addition, they reported a median OS benefit in patients with HCC treated with proton RT (31 vs 14 months), findings which are similar to those of the current study (33 vs 13 months). ${ }^{31,32}$ A prospective study of proton therapy for HCC and intrahepatic cholangiocarcinoma reported promising local control and OS rates as compared to historical data using photons, leading to a randomized study of protons versus photons for HCC (NRG GI003, NCT03186898). ${ }^{32}$ A trial at the University of Tsukuba reported that proton RT was both safe and effective for the treatment of HCC and demonstrated a 5-year OS of $44.5 \%{ }^{33}$ 
This study has some limitations. The small size of the proton cohort may limit robust statistical analysis to more completely characterize the impact of proton therapy on lymphocytes and survival outcomes. Additionally, as with any retrospective study, there may be an element of selection bias. In this study, venous tumor thrombosis also emerged as an independent prognostic factor. A greater proportion of patients treated with photons had portal venous tumor thrombosis and had Stage III or IV disease. Though these attributes were controlled for in our multivariable analysis, these differences may nonetheless confound interpretation of outcomes by technique. Patients who received proton RT in this group tended to have rightsided liver disease thereby resulting in reduced dose to the portal/splenic system. Lastly, splenic dose, which may affect the degree of lymphopenia, was not reported for the entire cohort as it was not routinely recorded prior to 2016. To mitigate this potential bias, a re-calculation of the splenic dose was performed in the matched cohort analysis; however, the sample size for this calculation was limited to 15 per cohort.

Given the hypothesis that RT-associated lymphopenia arises from the irradiation of circulating blood based on the results of this study, it will be important to examine the correlation between the time of delivery of RT and ALC. We hypothesize that reducing the time of exposure to RT and dose to the portal/splenic system may reduce the overall ALC decrease observed during radiation, and one strategy to achieve this may be the use of proton therapy given its dosimetric advantages over photon therapy. ${ }^{34}$ Monitoring ALC during RT as a potential predictive biomarker of outcome may allow clinicians to modify their management in order to produce better outcomes. Overall, our study demonstrates that lymphopenia in the peritreatment period is associated with poorer outcomes for patients with unresectable HCC. We have also shown that the use of proton therapy may be associated with mitigation of lymphopenia, reduced frequency of liver decompensation as measured by the change in CPS, and increased OS. These results indicate that the development of strategies to mitigate lymphopenia is warranted.

\section{Acknowledgments}

We gratefully acknowledge support from the Andrew Sabin Family Fellowship, the Sheikh Ahmed Center for Pancreatic Cancer Research, institutional funds from The University of Texas MD Anderson Cancer Center, the Khalifa Foundation, equipment support by GE Healthcare and the Center of Advanced Biomedical Imaging, Philips Healthcare, and Cancer Center Support (Core) Grant P30 CA016672 from the National Cancer Institute to MD Anderson. Dr. Eugene Koay was supported by the National Institutes of Health (1R01CA218004-01A1, 1R01CA221971-01A1, U54CA2 10181-01, U54CA143837 and U01CA196403). Preliminary analyses and portions of these data were presented at the 2017 Royal Australian and New Zealand College of Radiologists (RANZCR) Annual Scientific Meeting and the 2018 ASCO Gastrointestinal Cancers Symposium.

\section{Disclosure}

BD reports honorarium from Sermo, Inc. ACK reports ownership of shares in Aravive, Inc. SPN reports funding from the Australian Postgraduate Award, the Royal Australian and New Zealand College of Radiologists (RANZCR) Research Grants, and the Radiological Society of North America (RSNA) Fellow Grant. JMH reports research support from Oncosil, Galera, Auro, and Augmenix and is also a consultant for BTG, AbbVie, Celgene, AstraZeneca, BMS, and Varian. RT reports personal fees from QED Therapeutics/The Lynx Group. EBH reports grants from Merck. SK reports grants from NIH, DoD, and CPRIT. In addition, SK reports a patent for minibeam therapy. TSH reports consulting fees from Merck, Novocure, Synthetic Biologics, and research funding from Taiho, Astra-Zeneca, BMS, Puma, IntraOp, and Tesaro. SHL reports grants and personal fees from Beyond Spring Pharmaceuticals and Hitachi Chemical Diagnostics, as well as personal fees from AstraZeneca, Inc and STCube Pharmaceuticals. EJK reports grants from National Institutes of Health, Stand Up 2 Cancer, MD Anderson Cancer Center, Elekta, and GE Healthcare, as well as personal fees from RenovoRx and Taylor and Francis. All reported conflicts are outside the submitted work. The authors report no other conflicts of interest in this work.

\section{References}

1. Ghouri YA, Mian I, Rowe JH. Review of hepatocellular carcinoma: epidemiology, etiology, and carcinogenesis. J Carcinog. 2017;16:1. doi:10.4103/jcar.JCar_9_16

2. Shebl FM, Capo-Ramos DE, Graubard BI, McGlynn KA, Altekruse SF. Socioeconomic status and hepatocellular carcinoma in the United States. Cancer Epidemiol Biomarkers Prev. 2012;21 (8):1330-1335. doi:10.1158/1055-9965.EPI-12-0124 
3. SEER. Cancer stat facts: liver and intrahepatic bile duct cancer. Available from: https://seer.cancer.gov/statfacts/html/livibd.html. Accessed November 21, 2020.

4. Skinner HD, Hong TS, Krishnan S. Charged-particle therapy for hepatocellular carcinoma. Semin Radiat Oncol. 2011;21(4):278-286. doi:10.1016/j.semradonc.2011.05.007

5. Hall EJ, Wuu C-S. Radiation-induced second cancers: the impact of 3D-CRT and IMRT. Int J Radiat Oncol Biol Phys. 2003;56(1):83-88. doi:10.1016/S0360-3016(03)00073-7

6. Newhauser WD, Durante M. Assessing the risk of second malignancies after modern radiotherapy. Nat Rev Cancer. 2011;11(6):438-448. doi:10.1038/nrc3069

7. Fang P, Shiraishi Y, Verma V, et al. Lymphocyte-sparing effect of proton therapy in patients with esophageal cancer treated with definitive chemoradiation. Int J Part Ther. 2018;4(3):23-32. doi:10. 14338/IJPT-17-00033.1

8. Ellsworth SG. Field size effects on the risk and severity of treatment-induced lymphopenia in patients undergoing radiation therapy for solid tumors. Adv Radiat Oncol. 2018;3(4):512-519. doi:10. 1016/j.adro.2018.08.014

9. Shiraishi Y, Fang P, Xu C, et al. Severe lymphopenia during neoadjuvant chemoradiation for esophageal cancer: a propensity matched analysis of the relative risk of proton versus photon-based radiation therapy. Radiother Oncol. 2018;128(1):154-160. doi:10.1016/j. radonc.2017.11.028

10. Wild AT, Ye X, Ellsworth SG, et al. The association between chemoradiation-related lymphopenia and clinical outcomes in patients with locally advanced pancreatic adenocarcinoma. Am $J$ Clin Oncol. 2015;38(3):259-265. doi:10.1097/COC.0b013e31 $82940 \mathrm{ffg}$

11. Cho O, Chun M, Chang SJ, Oh YT, Noh OK. Prognostic value of severe lymphopenia during pelvic concurrent chemoradiotherapy in cervical cancer. Anticancer Res. 2016;36(7):3541-3547.

12. Fang P, Jiang W, Davuluri R, et al. High lymphocyte count during neoadjuvant chemoradiotherapy is associated with improved pathologic complete response in esophageal cancer. Radiother Oncol. 2018;128(3):584-590. doi:10.1016/j.radonc.2018.02.025

13. Campian JL, Sarai G, Ye X, Marur S, Grossman SA. Association between severe treatment-related lymphopenia and progression-free survival in patients with newly diagnosed squamous cell head and neck cancer. Head Neck. 2014;36(12):1747-1753. doi:10.1002/ hed. 23535

14. Tang C, Liao Z, Gomez D, et al. Lymphopenia association with gross tumor volume and lung V5 and its effects on non-small cell lung cancer patient outcomes. Int J Radiat Oncol Biol Phys. 2014;89 (5):1084-1091. doi:10.1016/j.ijrobp.2014.04.025

15. Cho O, Oh YT, Chun M, Noh OK, Lee HW. Radiation-related lymphopenia as a new prognostic factor in limited-stage small cell lung cancer. Tumour Biol. 2016;37(1):971-978. doi:10.1007/s13277015-3888-y

16. Mendez JS, Govindan A, Leong J, Gao F, Huang J, Campian JL. Association between treatment-related lymphopenia and overall survival in elderly patients with newly diagnosed glioblastoma. J Neurooncol. 2016;127(2):329-335. doi:10.1007/s11060-015-2037-1

17. Grassberger C, Ellsworth SG, Wilks MQ, Keane FK, Loeffler JS. Assessing the interactions between radiotherapy and antitumour immunity. Nat Rev Clin Oncol. 2019;16(12):729-745. doi:10.1038/ s41571-019-0238-9

18. Sung W, Grassberger C, McNamara AL, et al. A tumor-immune interaction model for hepatocellular carcinoma based on measured lymphocyte counts in patients undergoing radiotherapy. Radiother Oncol. 2020. doi:10.1016/j.radonc.2020.07.025
19. Llovet JM, Brú C, Bruix J. Prognosis of hepatocellular carcinoma: the BCLC staging classification. Semin Liver Dis. 1999;19 (3):329-338. doi:10.1055/s-2007-1007122

20. Grossman SA, Ellsworth S, Campian J, et al. Survival in patients with severe lymphopenia following treatment with radiation and chemotherapy for newly diagnosed solid tumors. J Natl Compr Canc Netw. 2015;13(10):1225-1231.

21. Deng W, Xu C, Liu A, et al. The relationship of lymphocyte recovery and prognosis of esophageal cancer patients with severe radiation-induced lymphopenia after chemoradiation therapy. Radiother Oncol. 2019;133:9-15. doi:10.1016/j.radonc.2018.12.002

22. Liu H, Wang $\mathrm{H}$, Wu J, et al. Lymphocyte nadir predicts tumor response and survival in locally advanced rectal cancer after neoadjuvant chemoradiotherapy: immunologic relevance. Radiother Oncol. 2019;131:52-59. doi:10.1016/j.radonc.2018.12.001

23. Byun HK, Kim N, Park S, Seong J. Acute severe lymphopenia by radiotherapy is associated with reduced overall survival in hepatocellular carcinoma. Strahlenther Onkol. 2019. doi:10.1007/s00066-01901462-5

24. Grassberger C, Hong TS, Hato T, et al. Differential association between circulating lymphocyte populations with outcome after radiation therapy in subtypes of liver cancer. Int $J$ Radiat Oncol Biol Phys. 2018;101(5):1222-1225. doi:10.1016/j.ijrobp.2018.04.026

25. Routman DM, Garant A, Lester SC, et al. A comparison of grade 4 lymphopenia with proton versus photon radiation therapy for esophageal cancer. Adv Radiat Oncol. 2019;4(1):63-69. doi:10.1016/j. adro.2018.09.004

26. Wild AT, Herman JM, Dholakia AS, et al. Lymphocyte-sparing effect of stereotactic body radiation therapy in patients with unresectable pancreatic cancer. Int J Radiat Oncol Biol Phys. 2016;94(3):571-579. doi:10.1016/j.ijrobp.2015.11.026

27. Gryziak M, Woźniak K, Kraj L, Stec R. Milestones in the treatment of hepatocellular carcinoma: a systematic review. Crit Rev Oncol Hematol. 2020;103179. doi:10.1016/j.critrevonc.2020.103179

28. Cho Y, Park S, Byun HK, et al. Impact of treatment-related lymphopenia on immunotherapy for advanced non-small cell lung cancer. Int J Radiat Oncol Biol Phys. 2019;105(5):1065-1073. doi:10.1016/j. ijrobp.2019.08.047

29. Chen D, Patel RR, Verma V, et al. Interaction between lymphopenia, radiotherapy technique, dosimetry, and survival outcomes in lung cancer patients receiving combined immunotherapy and radiotherapy. Radiother Oncol. 2020;150:114-120. doi:10.1016/j. radonc.2020.05.051

30. Ménétrier-Caux C, Ray-Coquard I, Blay J-Y, Caux C. Lymphopenia in cancer patients and its effects on response to immunotherapy: an opportunity for combination with cytokines? J Immunother Cancer. 2019;7(1):85. doi:10.1186/s40425-019-0549-5

31. Sanford NN, Pursley J, Noe B, et al. Protons vs photons for unresectable hepatocellular carcinoma: liver decompensation and overall survival proton radiotherapy for hepatocellular carcinoma. Int J Radiat Oncol Biol Phys. 2019. doi:10.1016/j.ijrobp.2019.01.076

32. Hong TS, Wo JY, Yeap BY, et al. Multi-institutional phase II study of high-dose hypofractionated proton beam therapy in patients with localized, unresectable hepatocellular carcinoma and intrahepatic cholangiocarcinoma. J Clin Oncol. 2016;34(5):460-468. doi:10.12 00/JCO.2015.64.2710

33. Nakayama H, Sugahara S, Tokita M, et al. Proton beam therapy for hepatocellular carcinoma: the University of Tsukuba experience. Cancer. 2009;115(23):5499-5506. doi:10.1002/cncr.24619

34. Wang X, Krishnan S, Zhang X, et al. Proton radiotherapy for liver tumors: dosimetric advantages over photon plans. Med Dosim. 2008;33(4):259-267. doi:10.1016/j.meddos.2007.04.008 


\section{Publish your work in this journal}

The Journal of Hepatocellular Carcinoma is an international, peerreviewed, open access journal that offers a platform for the dissemination and study of clinical, translational and basic research findings in this rapidly developing field. Development in areas including, but not limited to, epidemiology, vaccination, hepatitis therapy, pathology

Submit your manuscript here: https://www.dovepress.com/journal-of-hepatocellular-carcinoma-journal and molecular tumor classification and prognostication are all considered for publication. The manuscript management system is completely online and includes a very quick and fair peer-review system, which is all easy to use. Visit http://www.dovepress.com/ testimonials.php to read real quotes from published authors. 